\title{
Prevalence, Treatment Eligibility and Postoperative Survival for Europeans with Aortic Stenosis
}

\author{
Paolo De Sciscio ${ }^{1 *}$, Jacob Brubert ${ }^{2}$, Michele De Sciscio ${ }^{3}$, Marta Serrani ${ }^{2}$, Joanna Stasiak², Geoff D Moggridge ${ }^{2}$ \\ From World Society of Cardiothoracic Surgeons 25th Anniversary Congress, Edinburgh \\ Edinburgh, UK. 19-22 September 2015
}

\section{Background/Introduction}

Aortic Stenosis (AS) is the most frequent valvular pathology in the developed world. Whilst much is known about its pathogenesis and treatment, a paucity of data exists on the prevalence and number of patients eligible for valve replacement.

\section{Aims/Objectives}

To quantify prevalence, treatment eligibility and postoperative survival in patients with AS.

\section{Method}

A systematic search was conducted across PubMed and EMBASE for studies evaluating prevalence, severity, decision-making and survival in AS patients. Studies were selected using a priori defined criteria reviewed by two independent investigators. Prevalence rates $[95 \% \mathrm{CI}]$ were calculated and pooled using fixed and random-effects models (statistical heterogeneity evaluated via X2 and I2). Subsequently, Monte Carlo methods with beta distributions and 2012 population data were utilised to assess eligibility per treatment option (using ESC indications).

\section{Results}

Fifty-four studies were included encompassing 52,951 patients across 5 domains: prevalence, severity, symptom status, treatment and outcome. Pooled prevalence in the general population aged 55-74yrs and $>75 \mathrm{yrs}$ was $2.9 \%$ [1.5-4.3\%] and 13.6\% [8.3-18.9\%], respectively. Of these, 21.6\% [19.1-24.2\%] had severe AS with 71.1\% [62.779.4\%] symptomatic. SAVR was performed in $57.1 \%$ [47.7-66.6\%] of symptomatic patients and $28.2 \%$ [16.6$39.7 \%]$ of asymptomatic patients. In non-operated asymptomatic patients, $44.7 \%$ [36.7-53.0\%] progressed to
SAVR within 2yrs. Regardless of symptoms, SAVR was associated with a 3-fold increase in survival within $2 \mathrm{yrs}$ (OR: 3.6 and 3.9). In high-risk/inoperable severe symptomatic patients aged $\leq 75 \mathrm{yrs}, 39.9 \%$ [31.4-48.4\%] received TAVR. On Monte Carlo simulation, there are 9,187,586 [6,988,043-11,629,367] Europeans aged $\leq 55$ yrs with AS. Of these, 1,984,666 [1,494,584-2,550,594] have severe AS with $1,178,766[878,802-1,528,812]$ eligible for SAVR and 234,934 [149,699-340,960] eligible for TAVR.

\section{Discussion/Conclusion}

Within current ESC guidelines, approximately 1.2 million Europeans aged $\leq 55 \mathrm{yrs}$ are candidates for SAVR with an additional 230,000 patients aged $\leq 75 y$ rs eligible for TAVR.

\section{Authors' details \\ 'Department of Engineering, University of Cambridge, Trumpington Street, Cambridge CB21PZ, UK. ${ }^{2}$ Department of Chemical Engineering \& \\ Biotechnology, University of Cambridge, Pembroke Street, Cambridge, CB23RA, UK. ${ }^{3}$ Internal Medicine Service, Royal Adelaide Hospital, North Terrace, Adelaide 5000, Australia.}

Published: 16 December 2015

doi:10.1186/1749-8090-10-S1-A162

Cite this article as: De Sciscio et al:: Prevalence, Treatment Eligibility and Postoperative Survival for Europeans with Aortic Stenosis. Journal of Cardiothoracic Surgery 2015 10(Suppl 1):A162. 